\title{
Optimization of Liposomal Lipid Composition for a New, Reactive Sulfur Donor, and In Vivo Efficacy Studies on Mice to Antagonize Cyanide Intoxication
}

\author{
Ilona Petrikovics, ${ }^{1,2}$ Prashanth Jayanna, ${ }^{1,3}$ Jonathan Childress, ${ }^{1}$ \\ Marianna Budai, ${ }^{1,4}$ Sarah Martin, ${ }^{1}$ Galina Kuzmitcheva, ${ }^{1}$ and Gary Rockwood ${ }^{2}$ \\ ${ }^{1}$ Department of Chemistry, Sam Houston State University, Huntsville, TX 77341, USA \\ ${ }^{2}$ United States Army Medical Research Institute of Chemical Defense, Aberdeen Proving Ground, MD 21010, USA \\ ${ }^{3}$ Triesta Sciences, HealthCare Global Enterprises Ltd, P. Kalinga Rao Road, Sampangiramnagar, Bengaluru 560 027, India \\ ${ }^{4}$ Department of Pharmaceutics, Semmelweis University, Högyes Endre Street 7, H-1092 Budapest, Hungary
}

Correspondence should be addressed to Ilona Petrikovics, ixp004@shsu.edu

Received 8 August 2011; Revised 11 October 2011; Accepted 20 October 2011

Academic Editor: Guru V. Betageri

Copyright (C) 2011 Ilona Petrikovics et al. This is an open access article distributed under the Creative Commons Attribution License, which permits unrestricted use, distribution, and reproduction in any medium, provided the original work is properly cited.

Present studies have focused on a novel cyanide antidotal system, on the coencapsulation of a new sulfur donor DTO with rhodanese within sterically stabilized liposomes. The optimal lipid composition for coencapsulation of DTO with rhodanese is the combination of 1-palmitoyl-2-oleoyl-sn-glycero-3-phosphocholine, cholesterol, cationic lipid (DOTAP), and 1,2-dipalmitoyl-snglycero-3-phosphoethanolamine-N-[methoxy(polyethylene glycol)-2000] ammonium salt (with molar ratios of $82.7: 9.2: 3.0$ : 5.1). With the optimized compositions, prophylactic and therapeutic in vivo efficacy studies were carried out in a mice model. When DTO was coencapsulated with rhodanese and thiosulfate the prophylactic antidotal protection was $4.9 \times \mathrm{LD}_{50}$. Maximum antidotal protection against cyanide intoxication $\left(15 \times \mathrm{LD}_{50}\right)$ was achieved with coencapsulated rhodanese and $\mathrm{DTO} /$ thiosulfate in combination with sodium nitrite. When applied therapeutically, $100 \%$ survival rate (6/6) was achieved at $20 \mathrm{mg} / \mathrm{kg}$ cyanide doses with the encapsulated DTO-rhodanese-thiosulfate antidotal systems with and without sodium nitrite. These data are indicating that the appropriately formulated DTO is a promising sulfur donor for cyanide antagonism.

\section{Introduction}

The specific treatment of cyanide $(\mathrm{CN})$ intoxication means the use of scavengers (e.g., methemoglobin former sodium nitrite (SN) or cobalt compounds or cyanohydrin formers, hydroxocabalamin (Cyanokit has been approved in the US), cobinamide [1], and/or the conversion of $\mathrm{CN}$ to the less toxic thiocyanate $(\mathrm{SCN})$ with exogenously administered sulfane sulfur and sulfurtransferase enzymes [2-4]. Rhodanese (Rh) is the best characterized multifunctional, mitochondrial sulfurtransferase [5-8] catalyzing the transfer of a sulfane sulfur atom from a donor molecule to cyanide. Determining the exact role of nitrite in cyanide antagonism is not clearly understood yet. Earlier studies were focusing on the methemoglobin-forming effect of nitrite that act as a scavenger by forming a relative stable complex of cyanomethemoglobin $[3,4]$. Very recent studies are focusing on the mitochondria-linked mechanism of nitrite as a nitric oxide donor [9-11].

Extensive researches are also focusing on developing effective sulfur-containing compounds serving as sulfur donors for reacting with $\mathrm{CN}$ with or without $\mathrm{Rh}$. Thiosulfate (TS) is the classical sulfur compound found to participate in the enzyme reaction $[3,4,12]$. However, TS has limited ability to reach the endogenous $\mathrm{Rh}$ enzyme because of a nearly exclusive extracellular distribution [13]. Baskin et al., reported results on the efficacy of various sulfur donors demonstrating that altering the chemical substituent of the longer chain sulfide modified the ability of the candidate molecule to protect against $\mathrm{CN}$ toxicity [14]. 
Earlier investigations were focused on administration of free $\mathrm{Rh}$ and the sulfur donor (SD) directly into the bloodstream [15-18]. Unfortunately, the free Rh enzyme was rapidly destroyed by the body's immune system, which makes the efficacy of this approach quite limited. To overcome the limitations for the circulating free Rh, micro- or nanosized carrier systems among others sterically stabilized unilamellar liposomes of $\sim 100-150 \mathrm{~nm}$ diameter are in the focus of recent encapsulation efforts [19]. The encapsulation of Rh with a sulfur compound into liposomes-the so-called coencapsulation-can offer further advantages. Over stability enhancement for $\mathrm{Rh}$ the coencapsulation can provide better overall conversion of $\mathrm{CN}$, since the basis component for enzyme reaction, the sulfur donor no longer has to penetrate the liposome membrane.

The lipid composition has a significant impact on the encapsulation efficiency of the $\mathrm{Rh}$ and/or sulfur compound and on the in vivo stability and antidotal effect of the carrier system [19]. Thus, optimization of the liposomal composition is an inevitable step in the design of novel antidotal systems.

Present work deals with a new lipophilic sulfur-containing compound, developed at the US Army Medical Research Institute of Chemical Defense, called DTO. In order to achieve the highest $\mathrm{CN}$ antidotal protection, the liposomal encapsulation of DTO with and without Rh was examined.

The objectives of this study are (1) optimization of the liposomal encapsulation for the new sulfur donor, DTO, with superior sulfur donor reactivity to the present therapy TS; (2) in vivo efficacy study of the coencapsulated DTO with $\mathrm{Rh}$ in combination with sodium nitrite on mice.

\section{Materials and Methods}

All chemicals employed were of the highest purity commercially available: potassium cyanide, TS, sodium nitrite, phosphate buffer components, ethanol, sodium chloride, concentrated hydrochloric acid, and sodium hydroxide were purchased from J. T. Baker, (Phillipsburg, NJ), formaldehyde and ferric nitrate were purchased from Fisher Scientific (Pittsburgh, PA). The liposome components (1-palmitoyl-2-oleoyl-sn-glycero-3-phosphocholine (POPC), 1, 2-dioleoyl-sn-glycero-3-phosphocholine (DOPC), N-[1-(2,3dioleoyloxy)propyl]-N,N,N-trimethylammonium chloride (DOTAP), soy lecithin (LEC), cholesterol (Chol)) and Rh were purchased from Sigma-Aldrich (St. Louis, MO), and 1,2-dipalmitoyl-sn-glycero-3-phosphoethanolamine$\mathrm{N}$-[methoxy(polyethylene glycol)-2000] ammonium salt (PEG-PE-2000) was purchased from Avanti Polar Lipids (Alabaster, AL). Bio-Rad Protein Kit was purchased from Bio-Rad Life Sciences Laboratories, Hercules, CA.

2.1. Animals. Male (CD-1) mice (Charles River Breeding Laboratories, Inc., Wilmington, MA) weighing $18-20 \mathrm{~g}$ were housed at $21^{\circ} \mathrm{C}$ and in light-controlled rooms $(12 \mathrm{~h}$ light/dark, full-spectrum lighting cycle with no twilight), and were furnished with water and 4\% Rodent Chow (Teklad HSD, Inc., CITY, WI) ad libitum. All animal procedures were conducted in accordance with the guidelines by The Guide for the Care and Use of Laboratory Animals (National Academic Press, 1996), accredited by AAALAC (American Association for the Assessment and Accreditation of Laboratory Animal Care). At the termination of the experiments, surviving animals were euthanized in accordance with the 1986 report of the AVMA Panel of Euthanasia [20].

\subsection{Preparation of Sterically Stabilized Liposomes (SL) Encap-} sulating DTO (SL-DTO), or Rh (SL-Rh), or Both of Them (SL-DTO-Rh) with and without Coencapsulated TS. POPC, DOPC, DOTAP, LEC, PEG-PE-2000, and Chol dissolved in ethanol were applied in various molar ratios in order to determine the optimal lipid composition.

The liposomes were prepared by the thin-film hydration method [21]. DTO was codissolved with the lipids. As hydrating solution isotonic phosphate buffer $(\mathrm{pH}=7.4$; osmolarity $=290$ mosm) was added to the dry lipid films. Four different Rh concentrations $(0.25 \mathrm{mg} / \mathrm{mL}, 0.50 \mathrm{mg} / \mathrm{mL}$, $1.00 \mathrm{mg} / \mathrm{mL}$ and $1.67 \mathrm{mg} / \mathrm{mL}$ ), and four different DTO concentrations $(2.0 ; 10.0 ; 20.0$ and $30.0 \mathrm{mM})$ were investigated with various liposomal lipid compositions to evaluate the effects of these parameters on encapsulation efficiency for Rh and/or DTO. The total lipid concentration (lipids and Chol together) was $10.0 \mathrm{mg} / \mathrm{mL}$. In order to obtain a homogenous population of small unilamellar vesicles (SUV), the multilamellar vesicles (MLV) were extruded through polycarbonate filters (100 and $400 \mathrm{~nm}$ ) with an extruder (Avanti Polar Lipids Inc. Alabaster, AL). Extrusions were repeated five times for each membrane unless otherwise indicated.

2.3. Determination of Rhodanese Activity. The formation of SCN from $\mathrm{CN}$ was measured spectrophotometrically (Genesys 10UV, Thermo Electron Corporation, Waltham, MA) by the method of Westley [22], with minor modifications of Petrikovics et al. [23]. One unit of Rh was defined as the amount of enzyme that forms $1 \mu \mathrm{mol}$ of SCN in $1 \mathrm{~min}$.

2.4. Sulfur Donor Reactivity. Formation of SCN from CN with the investigated sulfur donors of TS and DTO were determined spectrophotometrically by the method of Westley [22] with minor modifications of Petrikovics et al. [23].

2.5. Optimal Rh Load for $S L-R h$. Four different Rh concentrations $(0.25 \mathrm{mg} / \mathrm{mL}, 0.50 \mathrm{mg} / \mathrm{mL}, 1.00 \mathrm{mg} / \mathrm{mL}, 1.67 \mathrm{mg} /$ $\mathrm{mL}$ ) were employed with a lipid composition of POPC: Chol: PEG-PE-2000 with and without DOTAP. Percentage of $\mathrm{Rh}$ incorporation within the liposomes was determined by the Bradford Assay [24].

2.6. Optimal Lipid Composition for Liposomal Rh Encapsulation. Optimal lipid composition for Rh encapsulation was determined based on the highest enzyme activity achieved by the same encapsulation process with various lipid compositions. Unencapsulated $\mathrm{Rh}$ was separated from SL-Rh by gel filtration on a G-100 Sephadex gel column $(0.7 \mathrm{~cm} \times 10 \mathrm{~cm}$; GE Healthcare BioSciences AB, Sweden $)$. 
Measurements were carried out in isotonic phosphate buffer at $\mathrm{pH}=7.4$. $\mathrm{Rh}$ activity for the fractions was determined as described above.

$$
\begin{aligned}
& \text { Encapsulation efficiency }(\%) \\
& \qquad=\frac{\text { activity of SL-Rh }}{\text { total Rh activity }} \times 100 .
\end{aligned}
$$

For the spectrophotometric assays, $50 \mu \mathrm{L}$ liposomal samples were used. All measurements were performed at least in triplicate.

2.7. Optimal Lipid Composition Determination for SL-DTO. The encapsulation efficiency for the sulfur donor DTO was determined by the $\mathrm{Rh}$ assay described above with constant $\mathrm{Rh}$ concentration. When Rh concentration was constant, the rate of formation of SCN was directly proportional to the sulfur donor concentration.

$$
\begin{aligned}
& \text { Encapsulation efficiency }(\%) \\
& \qquad=\frac{\text { concentration of encapsulated DTO }}{\text { total DTO concentration }} \times 100 .
\end{aligned}
$$

2.8. Optimal Lipid Composition Determination for SL-RhDTO. Formation of SCN by SL-Rh-DTO with various lipid compositions was measured spectrophotometrically as described above.

Encapsulation Efficiency (\%)

$$
=\frac{\mathrm{SCN} \text { formation by the given SL-Rh-DTO }}{\text { SCN formation by the original (before encapsulation) Rh and DTO concentration }} \times 100 \text {. }
$$

TABLE 1: Comparison of in vitro sulfur donor reactivity of TS and DTO determined with free Rh.

\begin{tabular}{lcc}
\hline \multicolumn{2}{c}{ Rate of CN conversion $(\mathrm{mmol} \mathrm{SCN} / \mathrm{min})$} & Ratio \\
\hline TS & DTO & DTO/TS \\
\hline 0.2 & 3.0 & 15.0 \\
\hline
\end{tabular}

mortality. Results are given as \% survival (animals alive/ animals total). Total numbers of animals were 6 for each therapeutic experiment for each antidotal system.

\section{Results and Discussion}

These studies focused on the encapsulation optimization for new sulfur donor DTO when encapsulated with Rh and/or TS within sterically stabilized liposomes. The in vitro sulfur donor reactivity comparison shows that DTO reacts 15times faster with $\mathrm{CN}$ at constant $\mathrm{Rh}$ concentration than TS (Table 1). Encapsulation efficiencies for both Rh and DTO were optimized as a function of Rh-load, DTO-load, and lipid composition.

When encapsulating $\mathrm{Rh}$ alone, small amount of the cationic lipid DOTAP proved to be beneficial to enhance encapsulation efficiency (Table 2). The optimum Rh concentration within the liposomes was $0.25 \mathrm{mg} / \mathrm{mL}$ (Table 2).

For the encapsulation of DTO with a concentration of $2 \mathrm{mM}$, six different liposomal compositions were examined to rule out the role of lipid composition (Table 3). Each contained PEG-PE-2000 in $5.1 \mathrm{~mol} \%$, lipid-to-Chol ratio was 9 to 1 . Also, the cationic lipid, DOTAP in $3.0 \mathrm{~mol} \%$, was utilized to influence the charge of the liposomal surface in hopes that a positively charged surface would provide increased affinity for DTO. On the basis of the results

it can be concluded that the presence of DOTAP leads
2.10. Therapeutic Protection against $C N$ in Mice Using SLDTO-TS and SL-DTO-TS-Rh in Combination with SN. Animals received antidotes administered intravenously one min after $\mathrm{CN}$ injection (sc). Doses of antidotes were the same as described above for the prophylactic experiments.

The animals were evaluated 24 hours after $\mathrm{CN}$ exposure for
2.9. Prophylaxis against $C N$ in Mice Using $S L-R h, S L-D T O$, SL-DTO-TS, SL-DTO-Rh, and SL-DTO-TS-Rh in Combination with $S N$. Experimental animals received $\mathrm{KCN}$ after pretreatment with antagonist(s) (sulfur donors and/or Rh and/or SN). Freshly prepared SL-DTO, SL-DTO-TS, SL-
DTO-Rh, and SL-DTO-TS-Rh were administered intravenously (iv) by tail vein injection to mice $10 \mathrm{~min}$ prior to receiving $\mathrm{CN}$ (sc). Using $10 \mathrm{~mL} / \mathrm{kg}$ doses of (SL-DTOexperiments 6 and 7) the animals received 15-20 units of Rh. Employing $10 \mathrm{~mL} / \mathrm{kg}$ injections of (SL-DTO; Table 4 4) the dose for DTO was $11.5 \mathrm{mg} / \mathrm{kg}$ and $14.2 \mathrm{mg} / \mathrm{kg}$ for the prior to $\mathrm{CN}$ (sc) injection (Table 4 experiments 4 and 7). mortality; surviving animals were observed for an additional could be late-developing toxicity. No toxic effects which SL-DTO-TS-Rh, TS, or SN (when administered alone or in various combinations) were noted in any of the mice at the up and down method [25], using 8-18 mice for each $\mathrm{LD}_{50}$ expressed as a ratio of $\mathrm{LD}_{50 \text { (mean) }}$ of $\mathrm{CN}$ with antagonists and $\mathrm{LD}_{50 \text { (mean) }}$ of $\mathrm{CN}$ without antagonists. 
TABLE 2: Rh-load optimization with and without DOTAP.

\begin{tabular}{lcc}
\hline Composition of liposomes & $\begin{array}{c}\text { Original Rh concentration } \\
(\mathrm{mg} / \mathrm{mL})\end{array}$ & $\begin{array}{c}\text { \% of Rh incorporation determined } \\
\text { by Bradford }\end{array}$ \\
\hline POPC $:$ Chol $:$ PEG-PE-2000 $=56.9: 38: 5.1$ & 1.00 & 16.6 \\
POPC $:$ Chol $:$ PEG-PE-2000 $=56.9: 38: 5.1$ & 0.25 & 26.9 \\
POPC $:$ Chol $:$ PEG-PE-2000 : DOTAP $=82.7: 9.2: 5.1: 3.0$ & 1.67 & 18.2 \\
POPC $:$ Chol $:$ PEG-PE-2000 : DOTAP $=82.7: 9.2: 5.1: 3.0$ & 0.50 & 55.8 \\
POPC $:$ Chol $:$ PEG-PE-2000 : DOTAP $=82.7: 9.2: 5.1: 3.0$ & 0.25 & 74.0 \\
POPC $:$ Chol $:$ PEG-PE-2000 : DOTAP $=82.7: 9.2: 5.1: 3.0$ & 1.00 & 52.9 \\
\hline
\end{tabular}

TABLE 3: Encapsulation efficiencies for DTO in various liposome compositions with and without DOTAP. DTO concentration was 2.0 mM. Total lipid concentration was $10.0 \mathrm{mg} / \mathrm{mL}$.

\begin{tabular}{lcccccc}
\hline & \multicolumn{2}{c}{ Liposomal composition (mol\%) } & \multicolumn{4}{c}{ Encapsulation efficiency (\%) with various lipid components } \\
Lipid & Chol & PEG-PE-2000 & DOTAP & POPC & DOPC & LEC \\
\hline 82.71 & 9.19 & 5.1 & 3.0 & $78.4 \pm 2.3$ & $81.7 \pm 3.1$ & $64.3 \pm 3.0$ \\
85.41 & 9.49 & 5.1 & - & $60.7 \pm 3.0$ & $63.8 \pm 1.2$ & $61.6 \pm 1.5$ \\
\hline
\end{tabular}

to significant $(P<0.05)$ higher encapsulation efficiency with DOPC and POPC as main lipid component. LEC liposomes demonstrated the least encapsulation efficiency for DTO, and DOTAP appeared to provide little to no enhancement (Table 3). The role of DOTAP in enhancing the encapsulation efficiency can be explained with electrostatic interactions between DOTAP and DTO at the $\mathrm{pH}$ value examined $(\mathrm{pH}=7.4)$. Early experiments with the $60: 40$ ratio of dipalmitoyl-phosphatidylcholine (DPPC) to Chol composition provided very low encapsulation efficiency for encapsulation of DTO (data not shown here). As DTO is a lipophilic sulfur donor, it can be expected that it is localized in the liposomal bilayer, more or less immersed in it. The saturated bonds of DPPC providing an ordered, relative rigid structure may inhibit the immersion of DTO in the liposomal membrane. Contrarily, POPC and DOPC possessing double bonds and as a consequent of it a less ordered and more fluid membrane structure can promote the encapsulation of DTO. Thus, instead of DPPC, DOPC, or POPC were used for liposome preparation and ratios were adjusted to $90: 10$ lipid to Chol. The results shown in Table 3 indicate that DOPC liposomes containing 3 mol\% DOTAP provided the highest encapsulation efficiency at $81.7 \pm 3.1 \%$. POPC liposomes with 3\% DOTAP were close behind with an encapsulation efficiency of $78.4 \pm 2.3 \%$. However, there was no significant difference between encapsulation efficiencies with DOPC and POPC.

The liposome compositions including DOTAP were used in further experiments due to the increase in encapsulation efficiency achieved by these films. The effect on encapsulation efficiency by the increase in DTO concentration was evaluated for DOPC and POPC containing liposome compositions with both sets of liposomes containing 3\% DOTAP. In order to evaluate the role of DTO concentration on the encapsulation efficiency each set's films were prepared with DTO concentrations of $10 \mathrm{mM}, 20 \mathrm{mM}$, and $30 \mathrm{mM}$.

The encapsulation efficiency remained high for each liposome formulation containing 3\% DOTAP for each applied
DTO concentrations of $10 \mathrm{mM}, 20 \mathrm{mM}$, and $30 \mathrm{mM}$. The encapsulation efficiencies of DTO for POPC samples were $69.7 \pm 2.3 \%, 82.8 \pm 7.1 \%, 79.2 \pm 8.1 \%$, while for the DOPC samples DTO encapsulation efficiencies of $74.2 \pm 2.0 \%$, $86.2 \pm 3.9 \%$, and $89.9 \pm 4.2 \%$ were determined, for $10 \mathrm{mM}$, $20 \mathrm{mM}$, and $30 \mathrm{mM}$ DTO concentrations, respectively. For a given DTO concentration there was no significant difference between the encapsulation efficiency values for DOPC or POPC liposomes $(P>0.05)$.

3.1. Reevaluation of Rhodanese Encapsulation. The optimal liposome composition for the encapsulation of $\mathrm{Rh}$ was determined in earlier experiments to be the 60:40 ratio of POPC to Chol [19]. However, the prior experiments did not evaluate the DOPC or the cationic lipid DOTAP. Furthermore, $\mathrm{Rh}$ was added either in isotonic HEPES buffer $(\mathrm{pH}=7.4-7.7)$ or in $5 \%(\mathrm{w} / \mathrm{w})$ aqueous solution of glucose (GLU; $\mathrm{pH}=4.2-7.8$ ) to the dry lipid films. For the purpose of coencapsulating DTO with Rh, the Rh encapsulation efficiency must be determined for the same lipid compositions and in the same hydrating systems as in the case of DTO. The optimal liposome composition for $\mathrm{Rh}$ encapsulation was the 90:10 ratio of POPC to Chol with the use of DOTAP. Also, the $3.0 \mathrm{~mol} \%$ DOTAP again increased the encapsulation efficiency for most of the different liposomal compositions (Table 2).

3.2. Coencapsulation of DTO and Rhodanese. For the coencapsulation of DTO and Rh, the combination of POPC, Chol, PEG-PE-2000, and DOTAP (with molar ratios of $82.7: 9.2: 5.1: 3.0$ ) was chosen as the most adequate liposome composition. The mentioned composition of sterically stabilized, positively charged liposomes performed the best in the coencapsulation, with a coencapsulation efficiency for Rh and DTO of $88.6 \pm 17.1 \%$ (with a Rh load of $0.25 \mathrm{mg} / \mathrm{mL}$ and a DTO concentration of $30 \mathrm{mM}$ ). As the coencapsulation efficiency was determined on the basis of SCN formation 
TABle 4: Prophylactic protection by various cyanide antidotal combinations. APR denotes antidotal potency ratio, which can be calculated as the ratio of the average $\mathrm{LD}_{50}$ of $\mathrm{CN}$ with and without antagonists.

\begin{tabular}{lccc}
\hline Exp no. & Treatment & $\mathrm{LD}_{50}(\mathrm{mg} / \mathrm{kg}$; mean; range) & APR \\
\hline 1 & Control & $7.8(4.6-13.1)$ & 1 \\
2 & (SL-DTO) (iv) $+\mathrm{CN}(\mathrm{sc})$ & $17.3(9.8-30.7)$ & 2.2 \\
3 & (SL-DTO-TS) (iv) $+\mathrm{CN}(\mathrm{sc})$ & $38.0(21.5-67.3)$ & 4.8 \\
4 & (SL-DTO-TS) (iv) + SN (ip) + CN (sc) & $52.7(29.7-93.2)$ & 6.7 \\
5 & (SL-DTO-Rh) (iv) + CN (sc) & $30.7(14.6-64.0)$ & 3.9 \\
6 & (SL-DTO-TS-Rh) (iv) + CN (sc) & $38.0(22.6-64.2)$ & 4.9 \\
7 & (SL-DTO-TS-Rh) (iv) + SN (ip) + CN (sc) & $120.0(68.2-213.0)$ & 15.3 \\
\hline
\end{tabular}

TABLE 5: Relative prophylactic antidotal potency ratios (RAPRs) to express enhancing effects by $\mathrm{Rh}, \mathrm{SN}$, and coencapsulated TS.

\begin{tabular}{cc}
\hline Enhancing effects by Rh & RAPR \\
Comparison of exps 5 and 2 & $3.9 / 2.2=\mathbf{1 . 8}$ \\
Comparison of exps 7 and 4 & $15.3 / 6.7=\mathbf{2 . 3}$ \\
\hline Enhancing effects by SN & RAPR \\
Comparison of exps 4 and 3 & $6.7 / 4.8=\mathbf{1 . 4}$ \\
Comparison of exps 7 and 6 & $15.3 / 4.9=\mathbf{3 . 1}$ \\
\hline Enhancement by coencapsulated TS & RAPR \\
Comparison of exps 3 and 2 & $4.8 / 2.2=\mathbf{2 . 1}$ \\
Comparison of exps 6 and 5 & $4.9 / 3.9=\mathbf{1 . 3}$ \\
\hline
\end{tabular}

by SL-Rh-DTO; the given value represents the combined effect of Rh and DTO in CN conversion. For the sake of comparison, encapsulation efficiency for the coencapsulated $\mathrm{Rh}$ alone-with $0.25 \mathrm{mg} / \mathrm{mL}$ concentration-was $74 \%$, while for DTO alone-with $10 \mathrm{mM}$ DTO load-was $57.7 \pm 8.1 \%$. Increasing the concentration of DTO produced similar encapsulation efficiencies, than in case of $10 \mathrm{mM}$. With DTO loads of $20 \mathrm{mM}$ and $30 \mathrm{mM}$ for the coencapsulated DTO encapsulation efficiencies of $55.6 \pm 4.0 \%$ and $61.6 \pm 17.6 \%$ were measured, respectively. The conversion of $\mathrm{CN}$ to $\mathrm{SCN}$ by the coencapsulation of $10 \mathrm{mM}, 20 \mathrm{mM}$, and $30 \mathrm{mM}$ DTO with $\mathrm{Rh}$ also proved to remain linear with an $R^{2}$ value of 0.9930. The ability to co-encapsulate DTO, or any other sulfur donor molecule with $\mathrm{Rh}$ should provide better overall conversion of $\mathrm{CN}$, since the sulfur donor no longer has to penetrate the liposome membrane.

3.3. In Vivo Efficacy Testing. In vivo evaluation of the optimized liposomal preparations made from DTO/(DTO + TS) and/or Rh were tested as cyanide antidotes on a mice model. Based on the above optimization efforts, for further in vivo evaluations we employed $3 \%$ DOTAP; $0.25 \mathrm{mg} / \mathrm{mL}$ $\mathrm{Rh}$ load, $30 \mathrm{mM}$ DTO load with the lipid composition of POPC : Chol : PEG-PE-2000: DOTAP $=82.7: 9.2: 5.1: 3.0$. The in vivo efficacy was expressed as antidotal potency ratio (APR).

The in vivo prophylactic treatment results with $\mathrm{Rh}$ and DTO/TS encapsulated within the optimized liposomal formulations are shown in Table 4.

SL-DTO alone provided a protection with an APR of 2.2. (Table 4 experiment 2). This protection was enhanced
TABLE 6: Therapeutic protection by various $\mathrm{CN}$ antidotal combinations. Control: $\mathrm{KCN} \mathrm{LD}_{50}=7.8(4.6-13.1) \mathrm{mg} / \mathrm{kg}$.

\begin{tabular}{lccc}
\hline Exp no. & $\begin{array}{c}\text { KCN dose } \\
(\mathrm{mg} / \mathrm{kg})\end{array}$ & Treatment & Survival (\%) \\
\hline 1 & 15 & (SL-DTO-TS-Rh) & $6 / 6(100 \%)$ \\
2 & 15 & (SL-DTO-TS) $+\mathrm{SN}$ & $6 / 6(100 \%)$ \\
3 & 15 & (SL-DTO-TS-Rh) $+\mathrm{SN}$ & $6 / 6(100 \%)$ \\
4 & 20 & (SL-DTO-TS) $+\mathrm{SN}$ & $4 / 6(67 \%)$ \\
5 & 20 & (SL-DTO-TS-Rh) & $6 / 6(100 \%)$ \\
6 & 20 & (SL-DTO-TS) & $3 / 6(50 \%)$ \\
7 & 20 & (SL-DTO-TS-Rh) $+\mathrm{SN}$ & $6 / 6(100 \%)$ \\
\hline
\end{tabular}

$(\mathrm{APR}=4.8)$ when TS was coencapsulated with DTO in a molar ratio of $1: 1$ (Table 4 experiment 3). Coencapsulation of TS with DTO is also believed to provide protection against product inhibition by sulphite (Zottolla, personal communication). As it was expected, SN further enhanced the protection with the APR of 6.7. (Table 4 experiment 4). When DTO was coencapsulated with Rh (Table 4 experiment 5), the APR was 3.9. When DTO was coencapsulated with TS and Rh, (SL-DTO-Rh) the APR was enhanced to 4.9 (Table 4 experiment 6$)$. The highest protection $(\mathrm{APR}=15.3$ ) was achieved with the combination of (SL-DTO-TS-Rh) and SN (Table 4 experiment 7). Expressing the relative antidotal potency ratios (RAPR) better indicates the differences in protection with two antidotal systems (Table 5). Comparing experiment 2 and $3(\mathrm{RAPR}=2.2)$ represents the effects of TS coencapsulation with DTO. When comparing experiments 3 and $4(\mathrm{RAPR}=1.4)$ or experiments 6 and $7(\mathrm{RAPR}=3.1)$ the enhancement effects reached by $\mathrm{SN}$ are represented. The significant enhancement by $\mathrm{Rh}$ is expressed when comparing experiments 2 and $5(\mathrm{RAPR}=1.8)$ and experiments 4 and 7 $($ RAPR $=2.3)$ confirming earlier studies with other types of encapsulation formulations for $\mathrm{Rh}[23,26,27]$.

Table 6 shows the therapeutic antidotal protection with the (SL-DTO-TS-Rh) combinations with and without SN. At approximately $2 \mathrm{LD}_{50}$ dose of $\mathrm{KCN}(15 \mathrm{mg} / \mathrm{kg})$, all the 6 animals survived in each experiment (Table 6 experiments 1,2 , and 3). However, when the KCN dose was enhanced $\left(20 \mathrm{mg} / \mathrm{kg}\right.$, approximately $\left.3 \mathrm{LD}_{50}\right)$ the survival rate with (SL-DTO-TS) + SN was 67\% (Table 6 experiment 4), while with (SL-DTO-TS) without SN provided a $50 \%$ survival rate (Table 6 experiment 6 ). However the (SL-DTO-TS-Rh) 
antidotal system with and without SN also provided a $100 \%$ therapeutic protection (Table 6 experiments 5 and 7).

\section{Conclusions}

The present experiments and results are confirming that the approach of utilizing externally administered, encapsulated, metabolizing rhodanese may have broad implications in cyanide antidotal therapy. The application of this approach has been successfully tested in animal models. In summary, these studies are describing the prophylactic and therapeutic in vivo efficacy of the encapsulated Rh and the new, reactive sulfur donor DTO. Optimization efforts were attempted for the liposomal lipid compositions, Rh-load, and coencapsulation of two sulfur donors (TS and DTO) and $\mathrm{Rh}$ to enhance the encapsulation efficiency for the given components. Optimization of the carrier systems is always a major part of these types of research efforts. Considering the high lipophilicity of DTO, for further in vivo applications other introduction routes (e.g., intramuscular) with further formulation optimizations are recommended.

\section{Acknowledgments}

This paper was supported by NIH: NIAID/USAMRICD Interagency Agreements (W911NF-07-D-0001), the USAMRICD under the auspices of the US Army Research Office Scientific Services Program administered by Battelle (Delivery order 0557, Contract no. TCN 08284). Many thanks for the support by the János Bolyai Research Scholarship of the Hungarian Academy of Sciences (M. Budai) and the Robert A. Welch Foundation (x-0011) at Sam Houston State University (Huntsville, Texas, USA).

\section{References}

[1] K. E. Broderick, P. Potluri, S. Zhuang et al., "Cyanide detoxification by the cobalamin precursor cobinamide," Experimental Biology and Medicine, vol. 231, no. 5, pp. 641-649, 2006.

[2] W. D. McGuinn, L. Baxter, L. Pei, I. Petrikovics, E. P. Cannon, and J. L. Way, "Antagonism of the lethal effects of cyanide by a synthetic water-soluble cobalt(III) porphyrin compound," Fundamental and Applied Toxicology, vol. 23, no. 1, pp. 76-80, 1994.

[3] J. L. Way, "Cyanide antagonism," Fundamental and Applied Toxicology, vol. 3, no. 5, pp. 383-386, 1983.

[4] J. L. Way, "Cyanide intoxication and its mechanism of antagonism," Annual Review of Pharmacology and Toxicology, vol. 24, pp. 451-481, 1984.

[5] J. Westley and D. Heyse, "Mechanisms of sulfur transfer catalysis. Sulfhydryl-catalyzed transfer of thiosulfonate sulfur," Journal of Biological Chemistry, vol. 246, no. 5, pp. 1468-1474, 1971.

[6] D. Bordo, D. Deriu, R. Colnaghi, A. Carpen, S. Pagani, and M. Bolognesi, "The crystal structure of a sulfurtransferase from Azotobacter vinelandii highlights the evolutionary relationship between the rhodanese and phosphatase enzyme families," Journal of Molecular Biology, vol. 298, no. 4, pp. 691704, 2000.

[7] T. P. Dooley, S. K. Nair, R. E. Garcia, and B. C. Courtney, "Mouse rhodanese gene (Tst): cDNA cloning, sequencing, and recombinant protein expression," Biochemical and Biophysical Research Communications, vol. 216, no. 3, pp. 1101-1109, 1995.

[8] Y. Saidu, "Physicochemical features of rhodanese: a review," African Journal of Biotechnology, vol. 3, no. 8, pp. 370-374, 2004.

[9] M. Otsuka, S. A. Marks, D. E. Winnica, A. A. Amoscato, L. L. Pearce, and J. Peterson, "Covalent modifications of hemoglobin by nitrite anion: formation kinetics and properties of nitrihemoglobin," Chemical Research in Toxicology, vol. 23, no. 11, pp. 1786-1795, 2010.

[10] L. K. Cambal, M. R. Swanson, Q. Yuan et al., "Acute, sublethal cyanide poisoning in mice is ameliorated by nitrite alone: complications arising from concomitant administration of nitrite and thiosulfate as an antidotal combination," Chemical Research in Toxicology, vol. 24, no. 7, pp. 1104-1112, 2011.

[11] L. L. Pearce, E. L. Manzano, S. Martinez-Bosch, and J. Peterson, "Antagonism of nitric oxide toward the inhibition of cytochrome c oxidase by carbon monoxide and cyanide," Chemical Research in Toxicology, vol. 21, no. 11, pp. 20732081, 2008.

[12] W. A. Himwich and J. P. Saunders, "Enzymatic conversion of cyanide to thiocyanate," American Journal of Physiology, vol. 153, pp. 348-354, 1948.

[13] P. Leung, L. E. Ray, C. Sander, J. L. Way, and D. M. Sylvester, "Encapsulation of thiosulfate: cyanide sulfurtransferase by mouse erythrocytes," Toxicology and Applied Pharmacology, vol. 83, no. 1, pp. 101-107, 1986.

[14] S. I. Baskin, D. W. Porter, G. A. Rockwood et al., "In vitro and in vivo comparison of sulfur donors as antidotes to acute cyanide intoxication," Journal of Applied Toxicology, vol. 19, no. 3, pp. 173-183, 1999.

[15] C. J. Clemedson, H. I. Hultman, and B. Sorbo, "The antidotal effects of some sulfur compounds and rhodanese in experimental cyanide poisoning," Acta Physiologica Scandinavica, vol. 32, pp. 245-251, 1954.

[16] A. Atkinson, D. A. Rutter, and K. Sargeant, "Enzyme antidote for experimental cyanide poisoning," Lancet, vol. 2, no. 7894, p. 1446, 1974.

[17] L. Frankenberg, "Enzyme therapy in cyanide poisoning: effect of rhodanese and sulfur compounds," Archives of Toxicology, vol. 45, no. 4, pp. 315-323, 1980.

[18] D. Weber, K. D. Friedberg, and L. Lendle, "Beurteilung therapeutisher Massnahmen bei der Blausaurevergiftung unter konstanter Cyanide-infusion. Naunyn-Schmiedebergs," Archiv für Experimentelle Pathologie und Pharmakologie, vol. 224, pp. $1-16,1962$.

[19] I. Petrikovics, M. Budai, S. I. Baskin et al., "Characterization of liposomal vesicles encapsulating rhodanese for cyanide antagonism," Drug Delivery, vol. 16, no. 6, pp. 312-319, 2009.

[20] "1986 report of the AVMA Panel on Euthanasia," Journal of the American Veterinary Medical Association, vol. 188, no. 3, pp. 252-268, 1986.

[21] D. Papahadjopoulos, T. M. Allen, A. Gabizon et al., "Sterically stabilized liposomes: improvements in pharmacokinetics and antitumor therapeutic efficacy," Proceedings of the National Academy of Sciences of the United States of America, vol. 88, no. 24, pp. 11460-11464, 1991.

[22] J. Westley, "Thiosulfate: cyanide sulfurtransferase (rhodanese)," Methods in Enzymology, vol. 77, no. C, pp. 285-291, 1981. 
[23] I. Petrikovics, E. P. Cannon, W. D. McGuinn et al., "Cyanide antagonism with carrier erythrocytes and organic thiosulfonates," Fundamental and Applied Toxicology, vol. 24, no. 1, pp. 86-93, 1995.

[24] M. M. Bradford, "A rapid and sensitive method for the quantitation of microgram quantities of protein utilizing the principle of protein dye binding," Analytical Biochemistry, vol. 72, no. 1-2, pp. 248-254, 1976.

[25] W. J. Dixon, "The up-and-down method for small animal samples," Journal of the American Statistical Association, vol. 12, pp. 967-978, 1965.

[26] E. P. Cannon, P. Leung, A. Hawkins, I. Petrikovics, J. DeLoach, and J. L. Way, "Antagonism of cyanide intoxication with murine carrier erythrocytes containing bovine rhodanese and sodium thiosulfate," Journal of Toxicology and Environmental Health, vol. 41, no. 3, pp. 267-274, 1994.

[27] I. Petrikovics, S. I. Baskin, K. M. Beigel et al., "Nano-intercalated rhodanese in cyanide antagonism," Nanotoxicology, vol. 4, no. 2, pp. 247-254, 2010. 

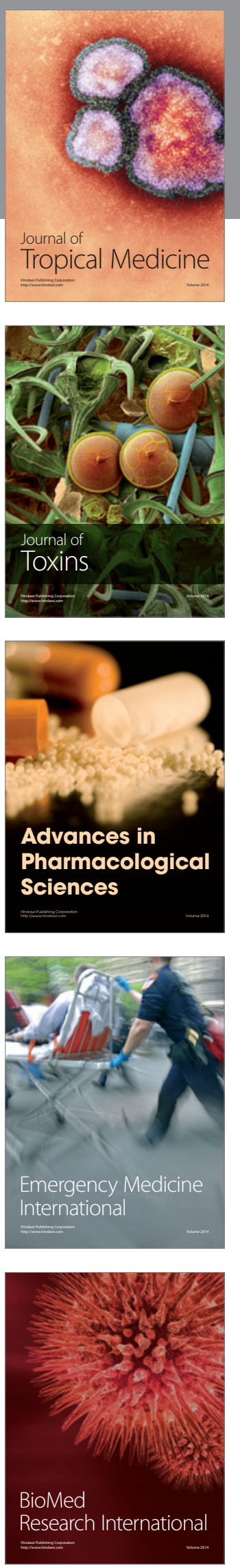
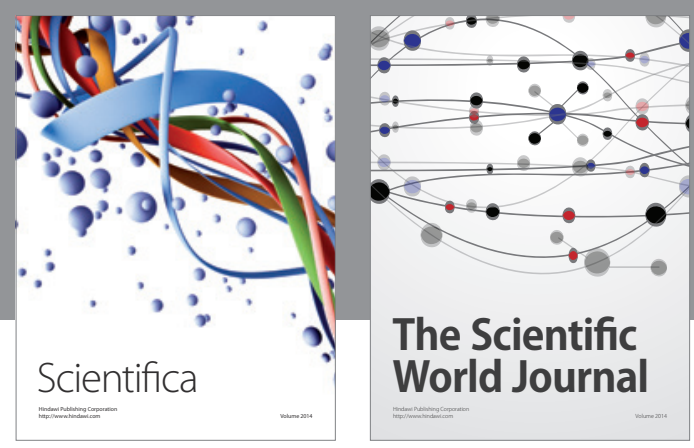

The Scientific World Journal
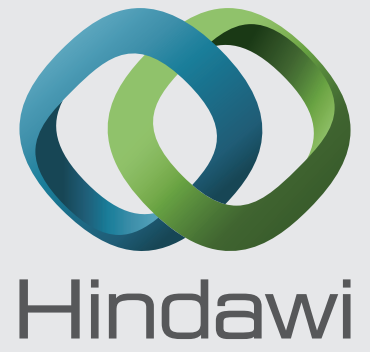

Submit your manuscripts at

http://www.hindawi.com
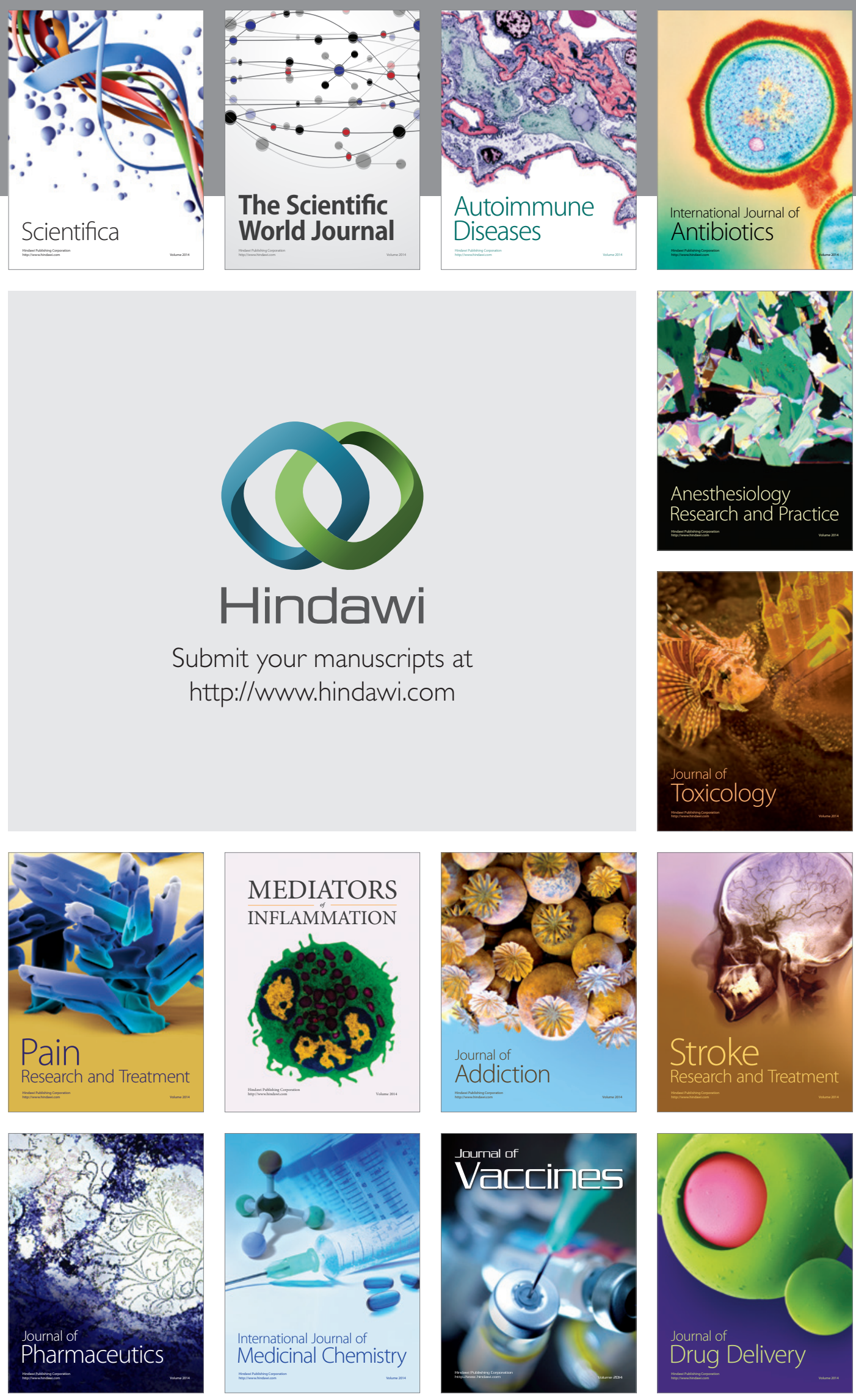